\title{
Sound-driven dissipative self-assembly of aromatic biomolecules into functional nanoparticles
}

Received 00th January 20xx, Accepted 00th January 20xx DOI: $10.1039 / x 0 x x 00000 x$

\author{
Sukhvir Kaur Bhangu ${ }^{a}$, Gianfranco Bocchinfuso ${ }^{c}$, Muthupandian Ashokkumar ${ }^{+}$and Francesca \\ Cavalieri ${ }^{b, c}+$
}

\begin{abstract}
The dissipative self-assembly processes were recently exploited to assemble synthetic materials into supramolecular structures. In most cases, chemical fuel or light driven self-assembly of synthetic molecules were reported. Herein, experimental and computational approaches were used to unveil the role of acoustic cavitation in the formation of supramolecular nanoaggregates by dissipative self-assembly. The acoustic cavitation bubbles were employed as an energy source and transient interface to fuel and refuel the dissipative self-assembly of simple aromatic biomolecules into uniform nanoparticles. Molecular Dynamics simulations was applied to predict the formation of metastable aggregates and the dynamic exchange of the interacting molecules in the nanoaggregates. The intracellular trafficking and dissipative dissolution of the nanoparticles were tracked by microscopy imaging.
\end{abstract}

\section{Introduction}

The dissipative self-assembly, DSA, processes are out-ofequilibrium ubiquitous phenomena observed in biological systems. For instance, the reversible formation of actin filaments is a case of dissipative self-assembly ${ }^{1}$. The products of these out-of-equilibrium biochemical reactions require a continuous supply of energy to persist, and their integrity is controlled by the relative rates of formation and disassembly driven by the energy dissipation processes ${ }^{2,3}$. Although common in biology, the DSA is only recently emerging as strategy for designing new functional materials with programmable lifetimes ${ }^{2-5}$.

Man-made precursor systems are turned into self-assembling building blocks by an activation reaction, triggered by a source of energy, typically provided by light or a fuel molecule ${ }^{2-8}$. The assemblies can only be maintained in their out-of-equilibrium state by a continuous input of energy or fuels that is subsequently converted to thermal energy and waste products. Chemical fuel and light driven DSA of organic and synthetic building blocks have been investigated, such as the selfassembly of vesicular nanoreactors ${ }^{9}$, the gelation of dibenzoylL-cysteine 10 , the light-driven azobenzene self-assembly into rod-like aggregates ${ }^{3}$ and the synthetic nucleic acid strands to

\footnotetext{
a. School of Chemistry, University of Melbourne, VIC 3010, Australia

b. Department of Chemical Engineering, University of Melbourne, VIC 3010,

Australia

c. Dipartimento di Scienze e Tecnologie Chimiche, Università di Roma "Tor Vergata", via della ricerca scientifica 1,00133, Rome, Italy

† E-mail: francesca.cavalieri@unimelb.edu.au.*E-mail: masho@unimelb.edu.au.

Electronic Supplementary Information (ESI) available: [Materials and Methods,

Characterization of dTrpNP-HPLC, EDS, Mass spectrometry, Fluorescence spectroscopy, Therapeutic properties of dTrpNPs.]. See DOI: 10.1039/x0xx00000x
}

design synthetic DNA-based receptors ${ }^{11}$. Strikingly, acoustic energy has not yet been considered as fuel in DSA processes. However, it was reported that low frequency sonication (29 and $80 \mathrm{kHz}$ ) can exert thermal and kinetic effects to promote the nucleation and the supramolecular reorganization of thermodynamically stable amyloid-like fibrils ${ }^{12}, 13$. In the latter study, low frequency ultrasound $(80 \mathrm{kHz})$ was used to trigger temporary supramolecular reconfiguration of assembled aromatic dipeptide amphiphiles from tapes to coiled fibers and straight fibers to spherical aggregates, which revert to the initial organization state when the sound is switched off.

To the best of our knowledge, the ultrasound-driven DSA has never been reported. Here, we provide an additional conceptual framework to obtain DSA of natural aromatic biomolecules. We combine experimental and computational approaches to unveil the role of the acoustic field in the formation of out-of-equilibrium nanoaggregates using, as a proof of concept, a simple amino acid, L-tryptophan. We anticipate that this approach can be used for the DSA of many other aromatic biomolecules, including phenylalanine, peptides, aromatic drugs and natural compounds.

We demonstrate that the acoustic bubbles driven at high frequency standing waves, provides a reactive surface for the dimerization of aromatic amino acids into amphiphilic molecules and an energy source to fuel and refuel their dissipative self-assembly into uniform nanoaggregates. The computational study predicts that the aggregation of tryptophan dimers occurs in less than 20 ns when a high local concentration is experienced at the cavitation bubble-solution interface. The lifetime of the nanoaggregates can be tuned by changing the $\mathrm{pH}$ of the media when the supply of acoustic energy is discontinued. The unique optical and bio-functional 


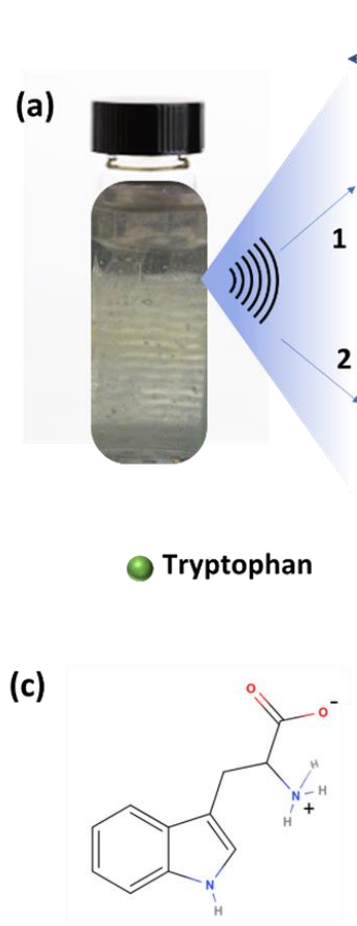

(1) Tryptophan

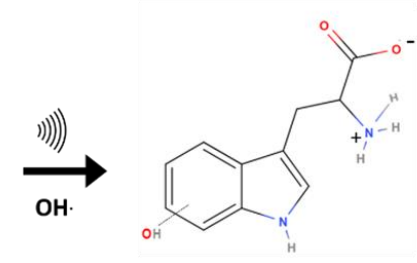

(2) $\sim 2 \mu \mathrm{s}$

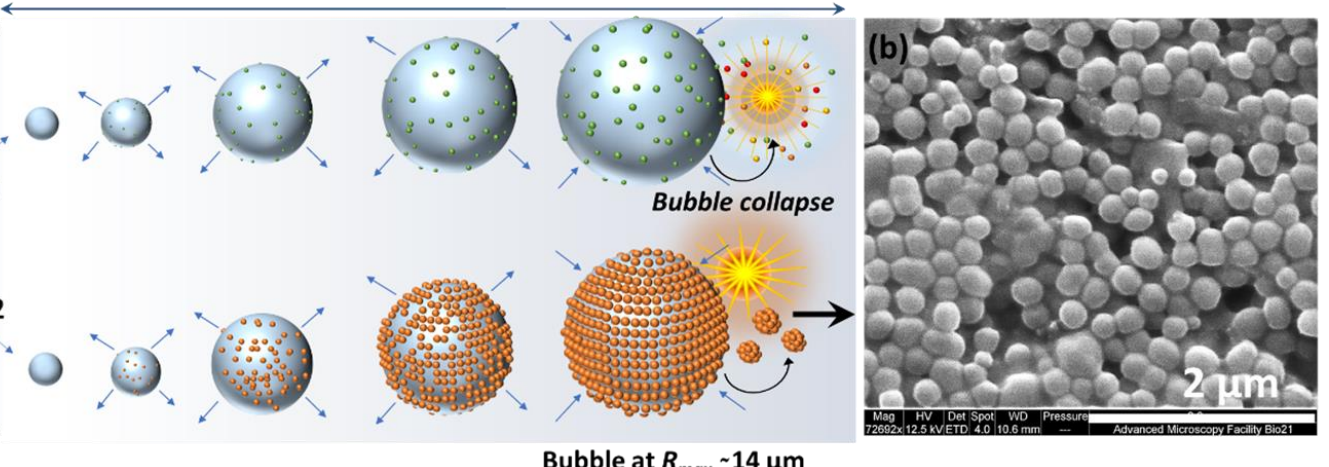

Bubble at $R_{\max } \sim 14 \mu \mathrm{m}$

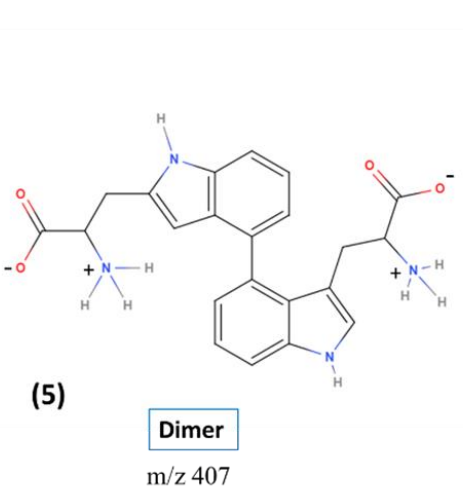

Hydroxytryptophan
$\mathrm{m} / \mathrm{z} 221$

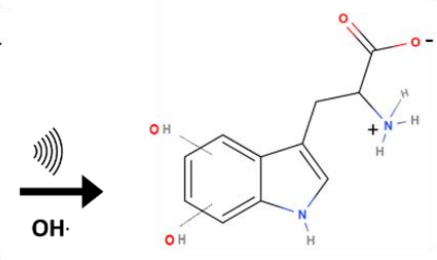

(3)

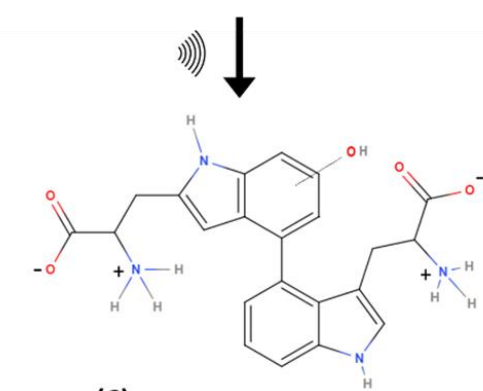

(6)

Hydroxylated Dimer $\mathrm{m} / \mathrm{z} 423$

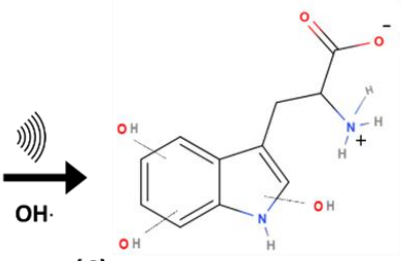

(4)

Tri-hydroxy tryptophan $\mathrm{m} / \mathrm{z} 253$

Figure. 1: Reaction scheme and proposed mechanism for the formation dTrpNPs by dissipative self-assembly (a) Schematic showing the formation of standing waves in the glass vial on sonication, increase in bubble size and gathering of Trp (step 1) and dTrp molecules (step 2) on bubble-solution interface during one oscillation cycle where collapse of the bubble generates hydroxylated products and dTrp (step 1) and dTrpNPs (step 2). (b) SEM images of the dTrpNPs generated after 3 h sonication of tryptophan below the cac (c) A reaction scheme showing the formation of the hydroxylated products due to attack of hydroxyl radicals on indole ring of tryptophan followed by dimerization reaction to form dTrp species which ultimately self-assemble into nanoparticles

properties of nanoparticles have been employed for probing their intracellular dissipative dissolution by imaging

\section{Results and Discussion}

The ultrasonic hydroxylation and dimerization of tryptophan.

To investigate the ability of ultrasound to induce tryptophan oxidation, dimerization and subsequently DSA of tryptophan dimers, an aqueous solution of tryptophan $(1 \mathrm{mg} / \mathrm{mL}, 4.9 \mathrm{mM}$, $\mathrm{pH}$ 5) was sonicated up to $5 \mathrm{~h}$ using ultrasonic frequency and power of $355 \mathrm{kHz}$ and $2 \mathrm{~W} / \mathrm{cm}^{2}$, respectively (see ESI Experimental Section). We selected $355 \mathrm{kHz}$ for our experiments because this is an optimal frequency to generate the required amount of $\mathrm{OH}$ radicals ${ }^{14}$. For instance in our experimental set up, we evaluated that after $1 \mathrm{~h}$ sonication $1 \mathrm{mM} \mathrm{OH}$ radicals are generated (see ESI, Experimental Section). The phenomenon occurring during sonication is depicted in Fig. 1a. We recently reported that the oscillating surface of acoustic cavitation microbubbles acts as a reactive and catalytic site for the $\mathrm{C}-\mathrm{C}$ coupling of amphiphilic moieties ${ }^{14}$. In this study, hydroxylated tryptophan species and hydroxylated tryptophan dimers, hereafter referred to as dTrp, were formed on the bubbles 
surface (Step 1 - Fig. 1a step 1 and Fig. 1c). The dTrp ultimately self-assembled into uniformly shaped spherical nanoparticles, dTrpNP, during bubbles collapse (Step 2 - Fig. 1b) with $40 \%$ yield. To gain insight into the chemical structure of oxidized tryptophan molecules and molecular composition of the nanoparticles, HPLC (ESI Fig. S1), mass spectrometry (ESI Fig. S2), EDS (energy dispersive X-ray spectroscopy (ESI Fig. S3a), FTIR (ESI, Fig. S3b), NMR (ESI Fig. S3c) and fluorescence spectroscopy (ESI Fig. S4) analyses were performed. The HPLC analysis (Fig. S1) shows the conversion of tryptophan (retention time $3.2 \mathrm{~min}$ ) into different products and the simultaneous appearance of two new peaks. The peak at retention time $4.4 \mathrm{~min}$ (Fig. S1b) was attributed to the dimers and hydroxylated dimers of tryptophan, whereas the peak at $2.7 \mathrm{~min}$ (Fig. S1c) was attributed to the hydroxylated tryptophan species. Mass spectrometry analysis performed on sonicated tryptophan solutions (Fig. S2a) confirmed the formation of hydroxylated products such as $\mathrm{Trp}+\mathrm{OH}, \mathrm{Trp}+2 \mathrm{OH}$ and $\mathrm{Trp}+3 \mathrm{OH}$ at $\mathrm{m} / \mathrm{z}$ of 221,237 and 253. In addition, different dimeric species with different degree of hydroxylation were also obtained (e.g. the peaks at $\mathrm{m} / \mathrm{z} 407$, $423,439)$. This indicates that both tryptophan or hydroxylated trytophan can combine to form dimers. The mass spectrometry analysis of dTrpNP (Fig. S2b), after purification and dissolution of nanopartcles, indicated that dTrpNP were mostly composed of monohydroxylated dTrp ( $\mathrm{m} / \mathrm{z}$ 445-dTrp). Correspondingly, the energy dispersive $\mathrm{X}$-ray spectroscopy of the dTrpNP confirmed an increase in the percentage of oxygen which could be attributed to the presence of hydroxyl groups (Fig. S3a). Fig. S3b shows the FTIR spectrum of dTrpNP in comparison with Trp, where peak at $1695 \mathrm{~cm}^{-1}$ corresponds to $\mathrm{C}=\mathrm{O}$ stretch, $1596 \mathrm{~cm}$ ${ }^{1}$ to $\mathrm{C}=\mathrm{C}$ aromatic stretch, $1324 \mathrm{~cm}^{-1}$ to $\mathrm{C}=\mathrm{C}$ indole stretch. The peak at $3400 \mathrm{~cm}^{-1}$ in $\operatorname{Trp}$ is due to $\mathrm{N}-\mathrm{H}$, however, a broad additional peak in dTrpNP at $3340 \mathrm{~cm}^{-1}$ corresponds to $\mathrm{O}-\mathrm{H}$ stretch. A comparison between the NMR spectra (Figure S3c) of unmodified tryptophan and dissolved dTrpNP indicate the absence of the peaks typically assigned to $\mathrm{H}-4$ aromatic proton. This can be explained by the dimerization of tryptophan via C-C coupling. The other aromatic peaks were observed in the region 6.9- $7.5 \mathrm{ppm}$. The presence of $\mathrm{ArOH}$ was confirmed by several peaks appearing around $8 \mathrm{ppm}$

Fig. S4a and S4b show the fluorescence emission and excitation spectra of sonicated tryptophan solution, respectively. We observed a red shift and an increase in emission intensity of sonicated tryptophan as a function of sonication time. The emission at $370 \mathrm{~nm}$ was ascribed to the formation of hydroxylated tryptophan species. This was confirmed by the excitation spectra showing a consistent decrease of the tryptophan peak at $280 \mathrm{~nm}$ with an increase in sonication time, and the appearance of a new peak at around $330 \mathrm{~nm}$ (Fig. S4b). The absorption spectra of the sonicated tryptophan solution acquired at different sonication times (Figure S4c) indicated the formation of species absorbing in the range of 300-600 nm When excited in the range $360-500 \mathrm{~nm}$, the sonicated solution exhibited an additional fluorescent peak at $465 \mathrm{~nm}$ ascribed to the dTrp. The extended degree of conjugation in the dTrp dimers lead to this emission peak. A shift in wavelenght from $420 \mathrm{~nm}$ to $570 \mathrm{~nm}$ was detected when excitation was changed from 360 to $500 \mathrm{~nm}$. This suggests the presence of multiple species which is in agreement with mass spectrometry analysis. Similar fluorescence emission in the range 400-600 nm was observed for cyclo-ditryptophan in self-assembled form but not in the soluble form ${ }^{16}$. The fluorescence properties of dTrpNPs suspension were also investigated (Fig. S5a) to confirm the chemical structure of the nanoparticles components. The fluorescence emission spectra of dTrpNPs show the characteristic peak of dTrp at $465 \mathrm{~nm}$, indicating the presence of dimers. Interestingly, intense emission bands in the far-red and near IR region (650-860 $\mathrm{nm}$ ) were observed when the dTrpNPs suspension was excited at $575 \mathrm{~nm}$ and $640 \mathrm{~nm}$ (Fig. S5a-b). As dTrp are not fluorescent in the near-red regions, the emission peaks can be ascribed to the nanoaggregates. These emissive states can arise from the aggregates as a result of the $\pi-\pi$ stacking interactions between aromatic moieties. In fact, when the fluorescence spectrum of dissolved dTrpNPs was acquired, the peaks in the red region were absent, whereas the emission of the soluble dTrp at $470 \mathrm{~nm}$ was preserved (Fig. S5a, green line), confirming that the red-shifted peaks at higher wavelengths $>600 \mathrm{~nm}$ are due to intermolecular interactions stabilizing the dTrpNPs. Taken together this comprehensive characterization study suggests the formation of different soluble hydroxylated Trp species where the dTrpNPs are made of less hydrophilic monohydroxylated dTrp. Figure 1c shows a schematic of the possible mechanistic pathways involved in the ultrasonic hydroxylation and dimerization of tryptophan. Previous computational and experimental studies have also shown that tryptophan bears multiple sites for the $\mathrm{OH}$ radical attack and hydrogen can also be abstracted from the indole rings ${ }^{17}$. It was shown that the hydroxylation of tryptophan by Fenton's reaction and photolysis oxidation occurs in different positions of the molecule yielding many possible isomers of hydroxytryptophans and monohydroxytryptophan dimers. ${ }^{21,22}$ However, none of these studies have ever reported the formation of nanoparticles ${ }^{21,22}$. It is well known that sonication of an aqueous solution results in the formation of $\mathrm{H}$ and $\mathrm{OH}$ radicals due to acoustic cavitation ${ }^{15}$. In our system, $\mathrm{OH}$ radicals generated during the high frequency ultrasonic treatment can abstract protons from the indole moiety which then undergo $\mathrm{OH}$ radical addition to form hydroxylated products (structure 2 , 3 and 4 in Figure 1c). In addition, two Trp radicals can combine through C-C coupling to form dimers or hydroxylated dTrp (structure 5, 6 and 7 in Figure 1c), where monohydroxylated dTrp self assemble into nanoparticles.

\section{The oscillation and collapse of transient cavitation bubbles} induce DSA of mono-hydroxytryptophan dTrp. 
(a)

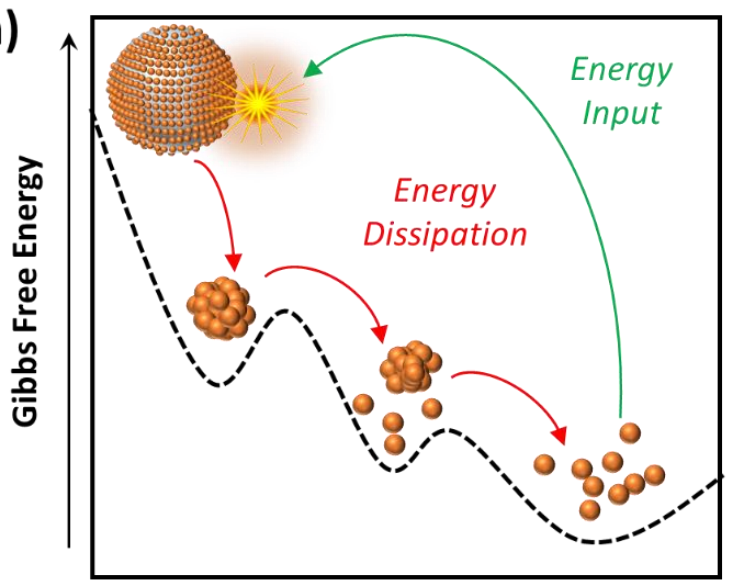

(b) 120

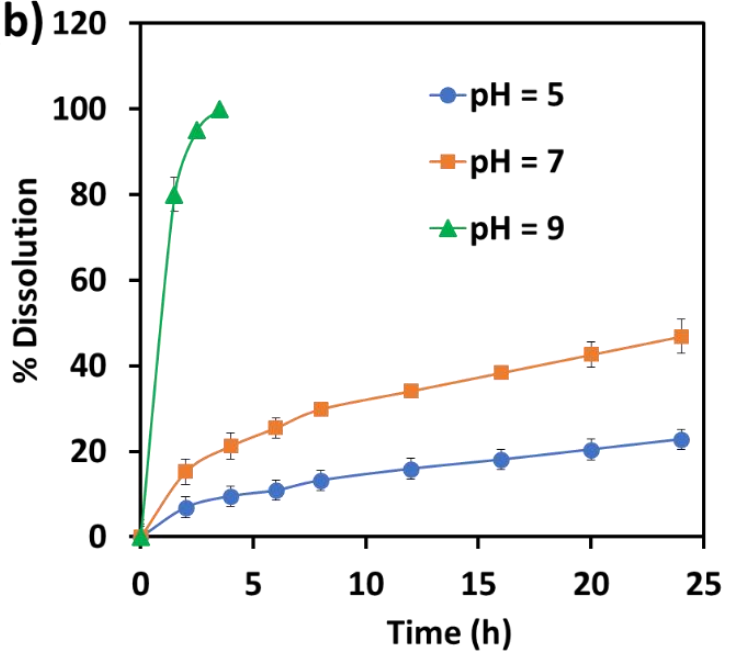

Energy

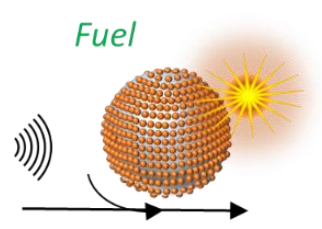

Dissipation

(c)

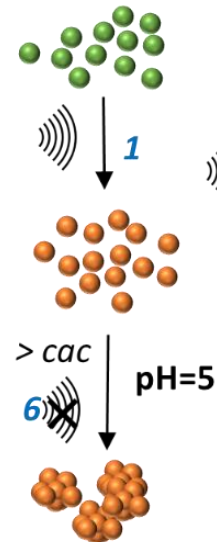

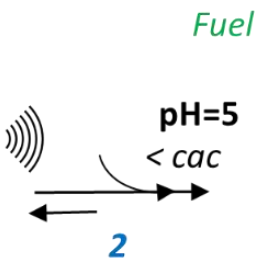
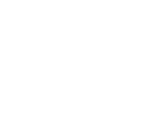

Tryptophan

- Building Blocks (dTrp)

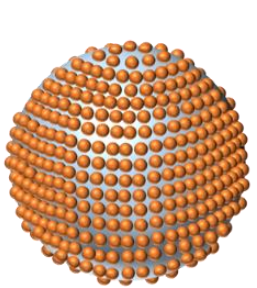

Template for aggregation

Figure 2: (a) The Gibbs free energy landscape of dissipative self-assembly of dTrp to form dTrpNPs where dTrp building blocks are residing in global minimum and transient energy input due to acoustic cavitation lead to out of equilibrium self-assembly of dTrp into dTrpNP. Furthermore, dTrpNPs undergo subsequent energy dissipation to recover to the original non-activated building block state over prolonged period of time ; (b) Dissolution kinetics of dTrpNPs at $37{ }^{\circ} \mathrm{C}$ at different $\mathrm{pH}$ as a function time suggesting slow dissolution of dTrpNP at $\mathrm{pH} 5, \mathrm{pH} 7$ and prompt dissolution at higher $\mathrm{pH}\left(\mathrm{pH}\right.$ 9) around the $\mathrm{pK}_{\mathrm{a}}$ of the aromatic hydroxyl and amine groups; (c) General scheme for the ultrasound driven dissipative self-assembly of dTrp to dTrpNPs. Step1 shows the ultrasonic conversion of Trp to building blocks dTrp, step 2 and 3 shows how the cavitation bubble acts as fuel providing a template for adsorption of dTrp and high concentration conditions to facilitate the aggregation upon collapse, leading to the formation dTrpNPs. Step 4 shows the energy dissipation step upon increasing $\mathrm{pH}$ to form the pristine building blocks, step 5 shows how the acoustic energy can refuel the dissipative self-assembly of dTrp below the cac at $\mathrm{pH} 2$.

The dynamic behavior of cavitation bubbles and the resulting physical and chemical effects are intertwined with the dissipative self-assembly of dTrp molecules as depicted in Fig. 1a. The change in bubble radius as a function of time (Fig. S6a) was calculated using Rayleigh-Plesset equation for the given frequency $(355 \mathrm{kHz})$ and power $\left(2 \mathrm{~W} / \mathrm{cm}^{2}\right) \cdot{ }^{15,18-20}$ From these data the maximum radius of gas bubbles driven by the acoustic field was estimated as $\sim 14 \mu \mathrm{m}$. Fig. S6a shows that a single acoustic cycle lasts for approximately $2 \mu \mathrm{s}$ at given conditions, during which the bubble size increases until the $R_{\max }$ is reached and bubble collapses. Based on the diffusion coefficient of the amino acids (700-1000 $\left.\mu \mathrm{m}^{2} / \mathrm{s}\right)$ and $\mathrm{dTrp}\left(870 \pm 78 \mu \mathrm{m}^{2} / \mathrm{s}\right)$ which was measured by fluorescence correlation spectroscopy (ESI Fig. S7), 14,18 this is likely a sufficient time for both Trp and dTrp molecules to diffuse, adsorb, dimerize and further aggregate during the bubbles collapse. First, the tryptophan molecules will be adsorbed at the bubble surface (Step 1-Fig.1a) to form hydroxylated and dimeric species mediated by bubbles collapse and generated $\mathrm{OH}$ radicals. Next, after $3 \mathrm{~h}$ sonication uniform spherical nanoparticles (dTrpNPs) were formed by the selfassembly of the dTrp species (Fig. 1a). In Fig. 1b, a representative SEM image of the uniform dTrpNP is shown. The average diameter of tryptophan nanoparticles obtained from the SEM analysis was $230 \mathrm{~nm} \pm 50 \mathrm{~nm}$. The $\zeta$-potential of dTrpNP measured at $\mathrm{pH} 7$ was found to be $-26 \pm 7 \mathrm{mV}$. dTrpNP were negatively charged because of the deprotonation of the carboxyl and aromatic hydroxyl groups in water at $\mathrm{pH}$ 7. This is supported by the potentiometric titration of dTrpNPs (ESI Fig. 
S8) after dissolution indicating three $\mathrm{pK}_{\mathrm{a}}$ values at approximately 2, 6 and 10 ascribed to carboxylic acid, hydroxyl and ammonium groups deprotonation respectively. Interestingly, we noticed that at these experimental conditions, the dTrp have a strong tendency to aggregate into nanoparticles only when ultrasound is applied. Conversely, after the rapid dissolution of dTrpNP in alkaline conditions at the same concentration as they were formed during sonication (50 $\mu \mathrm{g} / \mathrm{mL}, 1.6 \mathrm{mM})$, they were unable to self-aggregate, once the $\mathrm{pH}$ was brought back to neutral or acidic conditions. This indicates that the self-assembly of dTrp is thermodynamically disfavoured in bulk at that concentration and it is likely to be energetically fuelled by the high frequency ultrasound. To confirm this, the surface and aggregation properties of dTrp were evaluated. The surface tension of aqueous solution of $\mathrm{dTrp}$ dissolved at the same concentration (1.6 mM) was 67.3 $\mathrm{mN} / \mathrm{m}$ indicating surface activity and therefore, ability to adsorb on the cavitation bubbles. The critical aggregation concentration, cac, and the surface excess concentration (determined using Gibbs adsorption equation) of the $d \operatorname{Trp}$ were $100 \mathrm{\mu g} / \mathrm{mL}$ and $1.1 \times 10^{-6} \mathrm{~mol} / \mathrm{m}^{2}$, respectively. These results confirm that the ultrasound assisted self-assembly of dTrp occurred well below the cac $(50 \mu \mathrm{g} / \mathrm{mL})$. Hence, the association of dTrp building blocks to give aggregates is not thermodynamically favoured because the molecules reside in a global minimum in the energy landscape as depicted in Fig. 2 a.

(a)
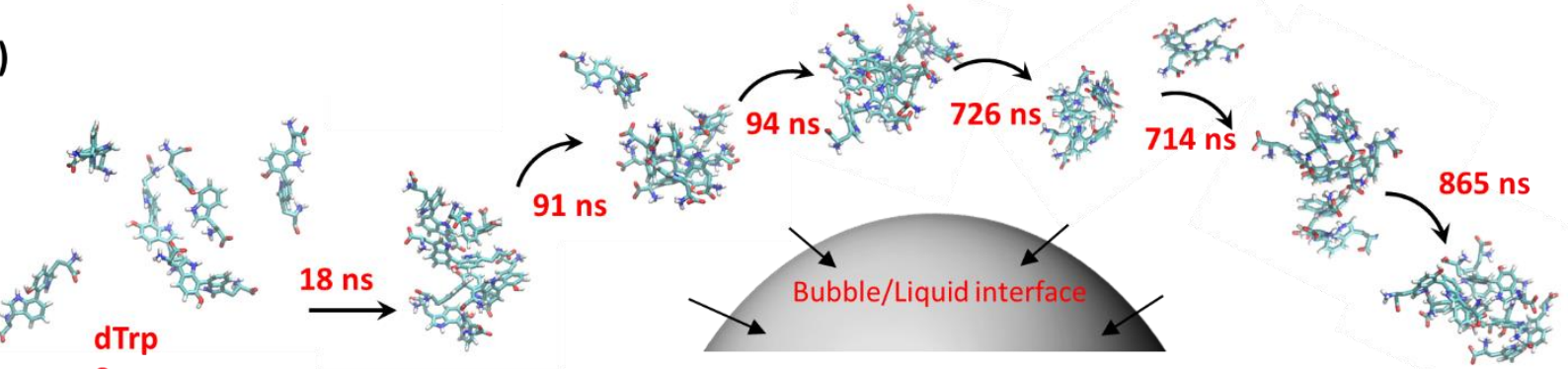

0 ns

(b)

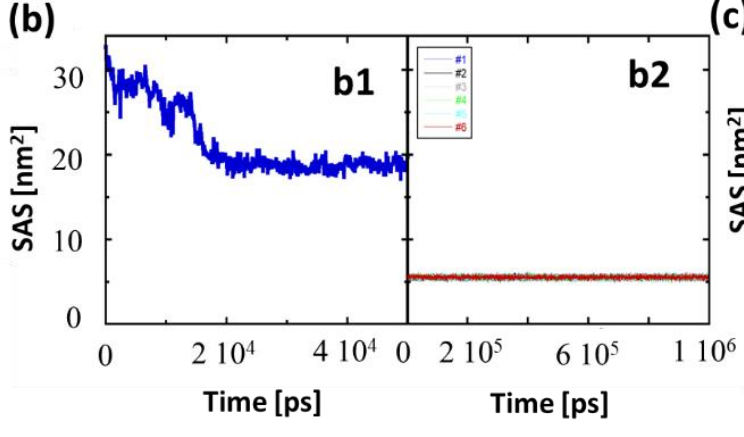

(c)

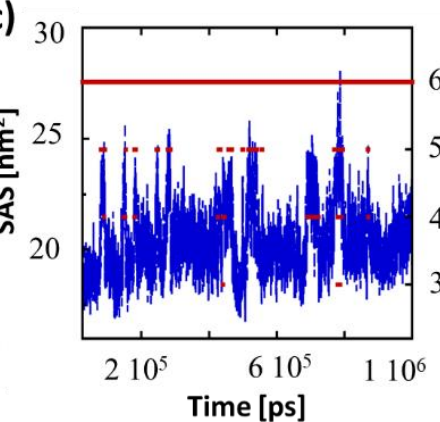

(e)

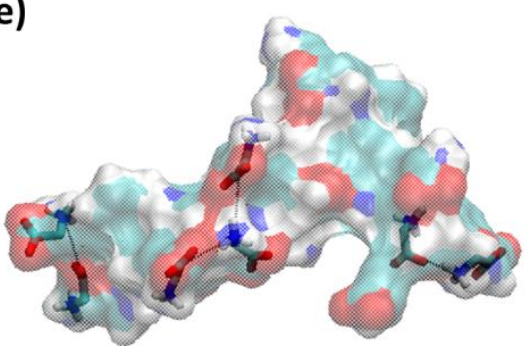

(f)

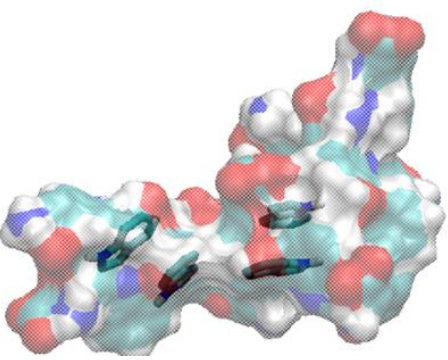

(d)

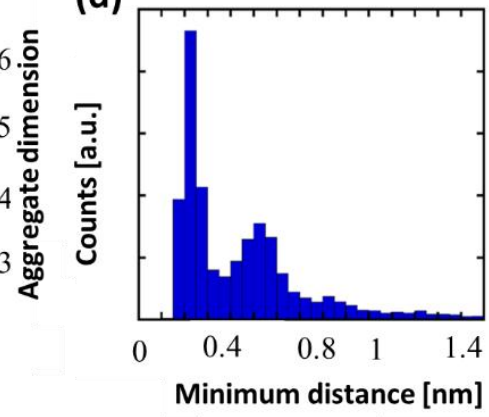

(g)

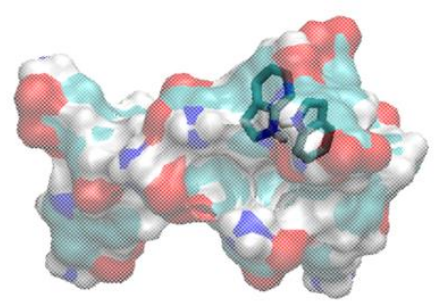

Figure 3: (a) Molecular dynamics simulation of six replicas of dTrp molecules, snapshots at 0 ns, 18 ns, 91 ns, 94 ns, 714 ns, 726 ns, and $868 \mathrm{~ns}$ and their schematic adsorption at bubble-liquid interface. The first aggregate was formed just after 18 $\mu \mathrm{s}$, (b1) Global SAS of the six dTrp molecules during the first 50 ns of the simulation, (b2) SAS calculated for all the six dTrp molecules singularly considered during the whole trajectory $(1 \mu \mathrm{s})$ (c) SAS value calculated on all the six dTrp molecules (blue line, $y$ axis on the left) and number of monomers in the aggregates (red points, y axis on the right) during the simulation time, (d) Distribution of the minimum distance of a pair of dTrp during the simulation. The distance has been calculated between 25 ns and $1 \mu s(e),(f)$ and (g) Snapshot from MD simulations. The dTrp molecule are shown as semitransparent surfaces. Models of $(e)$ ionic, $(f) \pi-\pi$ interactions and $(g) H$ bond involving aromatic rings as acceptors. The involved atoms are represented as solid sticks. The $C, H, N$ and $O$ atoms are colored in cyan, white, blue and red, respectively. 
However, a transient energy input provided by ultrasound (cavitation) can push the building blocks into a high-energy state where the self-assembly can take place (Fig.2a). The thermodynamically favoured dTrp nanoaggregates can be formed only at concentrations higher than cac and showed a different morphology with micrometer sized clusters which lack structural organization (Fig. S6b). The lifetimes of out of equilibrium dTrpNPs can be tuned by altering the kinetics of the dissipative step, i.e., by changing the pH (Fig.2b). dTrpNPs disassemble at $\mathrm{pH} 5$ over one week, dissolve within $48 \mathrm{~h}$ at $\mathrm{pH}$ 7 whereas dissolve within 5 hours by increasing $\mathrm{pH}$ above the $\mathrm{pK}_{\mathrm{a}}$ of the aromatic hydroxyl and amine groups ( $>\mathrm{pH} 10$ ) (Fig.2b). This indicates that the repulsive electrostatic interactions between negatively charged molecules can affect the kinetics of nanoparticle dissolution into water soluble building blocks.

We speculate on the possible role of cavitation bubbles in driving DSA processes. As shown in Fig.2c, the oscillating bubbles, driven by the high frequency acoustic field, provide a transient liquidair interface where dTrp are collected and preorganized (step 2 in Fig.2c). The experimental value of area occupied by each molecule on the gas-liquid interface was calculated using the surface excess measurements and it was found to be approximately $1.52 \mathrm{~nm}^{2}$. Assuming the molecules as spherical, the number of $d T r p$ covering the surface of oscillating bubbles at the maximum expansion (Step 2-Fig. 1c) can be approximately estimated as $2.4 \times 10^{8}$. In these transient conditions, the dTrp molecules experience an activated state and high local concentration. Upon bubbles collapse, the formation of uniform spherical nanoparticles far below the cac occur (step 3 in Fig.2c).

This is also energetically allowed because higher local concentration and strong intermolecular interactions are simultaneously experienced by the building blocks. Overall the dissipative self-assembly of dTrp is coupled to a chemical reactions network that includes (i) the irreversible chemical conversion of precursors (Trp) into building block (dTrp) (Step 1-Fig. 2c), (ii) the reversible activation of building blocks on the surface of cavitation bubbles which results in high local concentration of dTrp (Step 2 -Fig. 2c) and (iii) a reversible reaction where the activated building blocks self-assemble to form nanoaggregates (Step 3 -Fig. 2c). The nanoaggregates dissipate the free energy acquired during the steps (ii) and (iii) to recover the original non-activated building block state over prolonged time, depending upon the $\mathrm{pH}$ conditions (Step 4-Fig. 2c). The overall process has been described schematically in Fig. 2c. The nanoaggregates dissipate the energy and relax to the equilibrium state when the acoustic energy supply stops. This is because the nanoaggrates are out of equilibrium structures that return to the lower free energy, non-assembled state, mainly favored by the entropic terms. At any given $\mathrm{pH}$ the nanoaggregates relax spontaneously to the thermodynamic stable state over time (Step 4-Fig. 2c). However, in the presence of $\mathrm{OH}$ - the kinetics of dissolution of the nanoaggregates is faster.

Two assembly-disassembly cycles were performed to demonstrate that the system can be re-fuelled by the acoustic energy. The solution of dissolved dTrpNP was sonicated in acidic conditions at ultrasonic frequency and power of $355 \mathrm{kHz}$ and $2 \mathrm{~W} / \mathrm{cm}^{2}$ for $1 \mathrm{~h}$ to obtain nanoparticles. In the second cycle the protonation of carboxyl groups was required to assist the selfassembly of dTrp (Step 5- Fig. 2c). Fig. S9 a-b show the size distribution and SEM image of the nanoparticle obtained in second cycle. The Z-average size of nanoparticles was $\sim 188 \pm 50$ $\mathrm{nm}$. This may indicate that the amphiphilic properties of the building block changed upon sonication by further hydroxylation of dTrp molecules and the surface properties must be tuned by the protonation of carboxyl groups to maximize the surface activity and diffusion at the gas-liquid interface of dTrp. To support this hypothesis, the surface activity of dTrp in aqueous solution was determined using surface tension measurements at different $\mathrm{pH}$. The surface tensions at $\mathrm{pH} 2, \mathrm{pH} 7$ and $\mathrm{pH} 11$ were $58.9 \mathrm{mN} / \mathrm{m}, 70.5 \mathrm{mN} / \mathrm{m}$ and $80.7 \mathrm{mN} / \mathrm{m}$, respectively, suggesting higher surface activity of dTrp molecules at low $\mathrm{pH}$.

One can reasonably deduce that the sound driven self-assembly of dTrp is favoured when the carboxyl groups are protonated. It is worth mentioning that the cavitation bubble causes the dissociation of $\mathrm{N}_{2}$ and $\mathrm{O}_{2}$ present in air. The dissociated species and $\mathrm{N}_{2}$ and $\mathrm{O}_{2}$ can form $\mathrm{NO}_{x}, \mathrm{HNO}_{3}, \mathrm{HNO}_{2}$ and $\mathrm{HNO}$ which decrease the $\mathrm{pH}$ during the ultrasonic treatment to $\mathrm{pH} 2-3^{15}$. We noticed a shift in $\mathrm{pH}$ during the sonication from 5 to 2 in the first few hours of sonication when the precursors (Trp) convert into the building block (dTrp). This could explain why in the second cycle a pH adjustment of the solution is necessary to recover the original structure of the building block.Overall, our study indicates that acoustic cavitation is a fuel that provides both energy input and protons for self-assembly to take place.

Molecular Dynamics (MD) simulations predict the formation of metastable aggregates at high local concentration

To gain insight into the driving forces behind the formation of dTrpNPs and confirm the vital role of acoustic cavitation bubbles, Molecular Dynamics (MD) simulations of tryptophan dimers were performed. To this end, six replicas of dTrp were randomly inserted in a $64 \mathrm{~nm}^{3}$ cubic box in the presence of water Fig. 3a. This condition can mimic the high local concentration experienced by the amphiphilic dTrp molecules in proximity to the bubble collapse region (see Fig. 1a). MD simulations are usually unable to reproduce the spatial and the temporal scale required for analysis of larger aggregates, however, the early stages of aggregation can be simulated, and the data can be used to characterize the structural features of the final aggregates ${ }^{23}$. Representative snapshots from MD simulations up to $865 \mathrm{~ns}$ are shown in Fig. 3a. In Fig. 3b1, the Solvent Accessible Surface Area (SAS) of the six dTrp molecules during the first 50 ns of simulation is reported. The global SAS value of the solutes 
typically decreases when aggregation takes place, and this parameter is sensitive to both the number of the aggregates present in the simulation box and their compactness ${ }^{23,24}$. At the simulation start point, dTrp molecules are far from each other and the global SAS value is slightly below $33 \mathrm{~nm}^{2}$. Fig. $3 \mathrm{~b} 1$ shows that during the first 20 ns the SAS value of the six dTrp molecules decreases from 33 to roughly $20 \mathrm{~nm}^{2}$. The observed decrease in the global SAS is likely due to the occurrence of contacts between molecules upon aggregation. To confirm this, the SAS of each single dTrp molecule, during the whole simulation was evaluated (Fig. 3b2). In this case, the surface of each single dTrp molecule is considered as totally exposed to the solvent and its value could change only because of intramolecular conformational transition. Fig.3b2 shows that the starting SAS value of each single dTrp molecule is retained during the whole simulation, irrespective of presence or absence of aggregates. A value of $5.5 \pm 0.2 \mathrm{~nm}^{2}$ was measured for all the molecules, which is exactly a sixth of the starting value of the global SAS. This suggests that the intramolecular conformational changes only slightly affect the SAS of the single $\mathrm{dTrp}$ molecule and the global SAS variation is effectively probing the aggregation process. In addition, data reported in Fig. 3b1 indicate that, in the simulated conditions, stable aggregates form in less than $25 \mathrm{~ns}$. This lag time has been excluded from the further simulation analysis run up to $1 \mu \mathrm{s}$. Fig. $3 \mathrm{c}$ shows the behavior of the global SAS from $25 \mathrm{~ns}$ to the end of the simulation $(1 \mu \mathrm{s})$. From the minimum distances between all the possible pairs of monomers, we have evaluated at each time, the number of units present in the aggregates, i.e. aggregation number. Fig. $3 c$ shows that a single aggregate involving all the six molecules is present in almost all the simulation time (continuous red line). More in detail, aggregates of 6, 5, 4 and 3 $\mathrm{dTrp}$ molecules are present for the $87.6,8.1,4.2$ and $0.1 \%$ of the simulation time (dashed red lines) respectively. Figure $3 \mathrm{c}$ shows that when only an aggregate of six dTrp is present, the global SAS remains below roughly $22 \mathrm{~nm}^{2}$; on the other hand, when single monomers or dimers come off from the aggregate the SAS suddenly increases to higher value.

To discriminate the different contributions to the stabilization of the formed aggregates, the different interactions observed during the simulation were screened. The average number of hydrogen bonds detected during the simulations were $5 \pm 2$, however other interactions can play a role in the aggregate stabilization. Fig. 3e-g depicts the different interactions observed during the simulation. Ionic interactions between the charged carboxyl and amine groups have been first identified (Fig. 3e). The presence of aromatic moieties in aqueous environment favors the stabilization of $\pi-\pi$ interactions (Fig. 3f) between the indole moieties present in the dTrp molecules, which may result in peculiar optical features later investigated. Finally, in Fig. $3 g$ an example of unusual hydrogen bonds in which the aromatic ring act as acceptor is also reported. This kind of interaction, which is about half as strong as a normal hydrogen bond, is known to play a significant role in molecular associations $^{25}$. All these type interactions have been systematically observed throughout simulation process and we can assume they all contribute to the formation and stabilization of the nanoaggregate.

To investigate the persistence of the interactions between couples of molecules in the aggregate, we have calculated the distribution of the minimum distances for all the possible 15 pairs of dTrp molecules during the simulations. All distributions are similar, Fig.3d reports an example of this distribution profiles. The distribution shows that the pairs populate both low and high minimum distances values during the simulation. This suggests that, although the six molecules aggregate persists for almost all the simulation, the molecules assembled into the aggregate are dynamically interacting with each other. Overall the computational results are consistent with the rapid formation and stability of dTrp nanoparticles via salts bridges, $\boldsymbol{\pi}-\boldsymbol{\pi}$ and $\mathrm{H}$-bonding interactions. In addition, the dynamic exchange of the interacting molecules in the aggregates may contribute to the dissipation of energy process resulting in the dissolution of the nanoaggregates.

Finally, from the MD data we have evaluated the number of dTrp molecules present in a single $230 \mathrm{~nm}$ sized nanoparticle. From the six dTrp molecules aggregates SAS value $\left(20 \pm 1 \mathrm{~nm}^{2}\right)$ and assuming a spherical shape for the formed aggregates, we can estimate a volume of $8.4 \mathrm{~nm}^{3}$. As a result, the estimated number of dTrp molecules present in a single $230 \mathrm{~nm}$ sized nanoparticle are approximately $5 \times 10^{6}$. From the SAS data of isolated dTrp molecules (surface and planar area of to $5.5 \pm 2$ $\mathrm{nm} 2$ of $1.5 \mathrm{~nm}^{2}$ respectively), we can also estimate the maximum number of dTrp molecules that can be placed into a monolayer covering the whole surface of a microbubble which is approximately $245 \times 10^{6}$. This is in good agreement with the experimentally measured surface excess concentration (2.4 $\left.x 10^{8}\right)$. These results suggest that the collapse of a single bubble can in principle produces a single or more NPs. As the number of dTrp molecules covering the bubble surface exceeds the estimated number of molecules present in a single dTrpNP we can speculate that each collapsing bubble can give rise to the formation of a few nanoparticles as show in Figure 1a. In conclusion, the experimental and computational results are consistent with the hypothesis that the dTrpNPs arise from the rapid collapse of the microbubble and that they are formed by DSA of the molecules adsorbed at the surface of the same microbubble during the acoustic cavitation. The reported outof equilibrium system can be classified as DSA system because (i) it is fuelled and sustained by the input of acoustic energy and associated $\mathrm{pH}$ change, (ii) the nanoaggregates have finite lifetime and relax spontaneously to the thermodynamic stable state over time, when the fuel is removed (iii) the system can be refuelled by the acoustic energy and protons to promptly regenerate the nanoparticles.

\section{Probing the intracellular disassembly processes of dTrpNPs in different cellular compartments by imaging.}


A key challenge however, is understanding the interactions of dTrpNPs with biological systems at the cellular and subcellular levels. The kinetics and mechanism of cellular uptake, intracellular trafficking, dissolution and ultimately bioactivity of dTrpNPs strongly depends on whether the kinetics of disassembly of dTrpNPs investigated in test tube can take place in the crowded and complex intracellular milieu. We found that the optical properties of dTrpNPs offer a tool to gain insight into their intracellular behavior. dTrpNPs showed a good photostability under irradiation and at room T (Fig. S10a,b) and quantum yield $\left(Q Y_{575}=0.39\right)$. On the other hand, fluorescence probes like Rhodamine 6G, FITC, Cy3, etc. undergo bleaching in few seconds and require storage at $-20{ }^{\circ} \mathrm{C} 26-28$. These spectral features can be advantageous to track their intracellular trafficking and disassembling. The colloidal stability and integrity of dTrpNPs in the presence of serum proteins (100\% FBS ) was investigated. using Nanoparticle Tracking Analysis. A slight increase in particle size from $230 \pm 50 \mathrm{~nm}$ to $280 \mathrm{~nm} \pm 65$ $\mathrm{nm}$ was likely induced by adsorption of protein corona, wheras no aggregation or disassembly of dTrpNPs was observed. onto the surface of nanoparticles. Interstingly, the adsorption of protein corona on dTrpNPs surface produce a 3-fold increase in green emission (ESI Fig. S10c). This increase in emission was also observed when dTrpNPs were suspended in alkaline conditions (ESI Fig. S10d), therefore it was ascribed the deprotonation aromatic hydroxyl groups upon protein adsorption.

Next, we verified possible cellular cytotoxicity effects exerted by dTrpNPs. The MDA-MB-231 cells were incubated with dTrpNP at different concentrations from 3 to $100 \mu \mathrm{g} / \mathrm{ml}$ for 24 $\mathrm{h}$ and $48 \mathrm{~h}$. Fig. S11a shows that the particles exhibit negligible cytotoxicity even after $24 \mathrm{~h}$ and $48 \mathrm{~h}$ at all the tested concentrations. We evaluated the association of dTrpNPs with MDA-MB-231 cells as a function of time using flow cytometry under different fluorescent channels (Fig. S11b). The association relies on both membrane binding and intracellular uptake processes. We observed rapid and complete association of dTrpNPs with cells in the first $6.5 \mathrm{~h}$ of incubation (Fig. S11b). Furthermore, to study the uptake of dTrpNP, cells were incubated with $10 \mu \mathrm{g} / \mathrm{mL}$ nanoparticle suspension for $5 \mathrm{~h}$ and media was replaced followed by further incubation for up to 8 $\mathrm{h}, 24 \mathrm{~h}$ and $48 \mathrm{~h}$ at $37^{\circ} \mathrm{C}$ in fresh media.

The imaging of live cells incubated with dTrpNPs was carried out using confocal microscopy to investigate the kinetics of disassembly of dTrpNPs inside the cells. Fig. 4c shows blue, green and red fluorescence under different fluorescence channels. After $8 \mathrm{~h}$ and $24 \mathrm{~h}$ incubation, cells show mainly the punctuate fluorescence pattern with limited fluorescence diffusely spread throughout the cytosol in the blue, green and red channel. After $48 \mathrm{~h}$ incubation the cells exhibited blue and green fluorescence signal spread in the cytosol whereas the red fluorescence disappeared. The punctuate pattern indicates the partial confinement of dTrpNPs into acidic endo-lysosomes $(\mathrm{pH}$
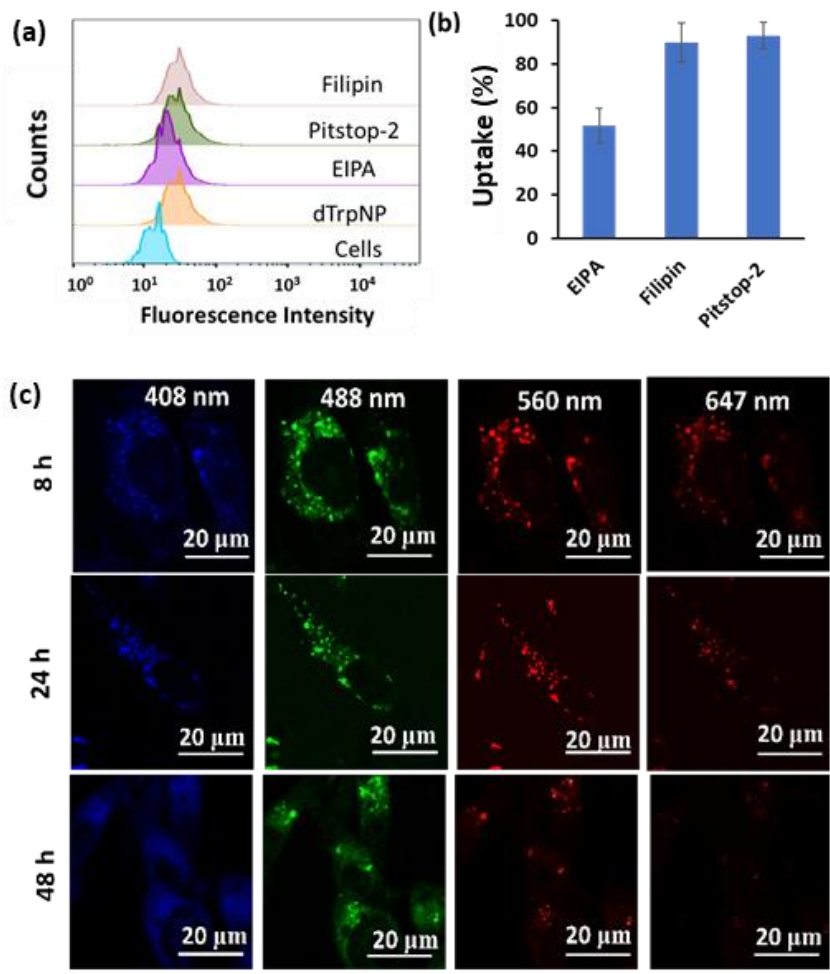

(d)
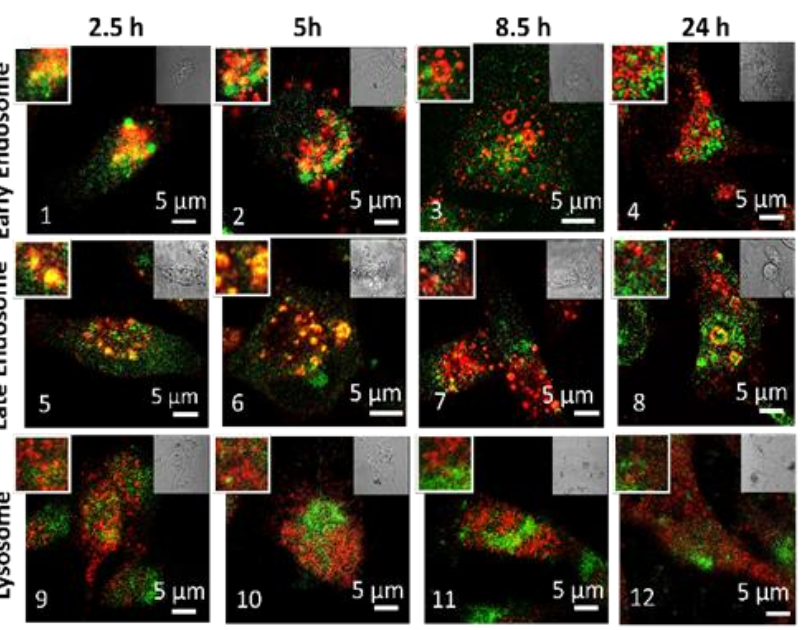

Figure 4: (a) Fluorescence intensity histogram of MDA-MB-231 cells before and after treatment with endocytic inhibitors and dTrpNPs measured using flow cytometry, (b) The effect of different endocytic inhibitors on the uptake of dTrpNP, (c) Confocal microscopic images of the MDA-MB-231 cells incubated with the dTrpNPs at $37^{\circ} \mathrm{C}$ for $8 h, 24 h$ and $48 h$; (d) Colocalization studies of dTrpNPs (green) with different constructs(red) after $2.5 h$ washout and further $5 h, 8 h$ and 24 $h$ incubation with confocal microscopy. The early endosome, late endosome and lysosome were stained with rabbit anti-EEA1 monoclonal antibody, rabbit anti-Rab7 monoclonal antibody and rabbit anti-LAMP-1 respectively followed by staining with the anti-rabbit secondary Alexa Fluor-647 conjugated antibody. Insert left zoomed image and insert bright field image of the principal image. 
5-6). As the red emission is indicative of the dTrpNPs aggregate state, these results suggest that a time dependent dissolution of dTrpNPs occurs in the cytosol $(\mathrm{pH} 7)$ after escaping from endo-lysosomes.

To further gain insight into cell internalization mechanism, MDA-MB-231 cells were incubated with filipin, pitstop-2, and ethylisopropyl amiloride (EIPA) to inhibit caveolae-dependent endocytosis, clathrin-dependent endocytosis and macropinocytosis respectively, and then incubated with dTrpNP $(10 \mu \mathrm{g} / \mathrm{ml})$. Fig. $4 \mathrm{a}$ and $4 \mathrm{~b}$ suggests that almost no uptake inhibition was observed with filipin and pitstop-2, however, EIPA led to $50 \%$ inhibition of uptake of dTrpNP. Therefore, the uptake of $d T r p N P$ can be mediated through macropinocytosis. To advance the understanding of the intracellular route of dTrpNPs, we examined their endocytic trafficking as a function of time in fixed cells by using the immunostaining of organelles. The MDA-MB-231 cells were incubated with dTrpNPs for $2.5 \mathrm{~h}$, washed with fresh medium to remove the extracellular dTrpNPs, and cultured for further $2.5 \mathrm{~h}, 5 \mathrm{~h}$ and $21.5 \mathrm{~h}$ at $37{ }^{\circ} \mathrm{C}$ corresponding to observation after $2.5 \mathrm{~h}, 5 \mathrm{~h}, 8.5 \mathrm{~h}$ and $24 \mathrm{~h}$. Immunostaining of early endosome, late endosome and lysosome was performed using EEA1 antibody, Rab7 antibody, LAMP 1 markers and AF 647 secondary antibody. Fig. 4d shows representative confocal microscopy images of cells vesicles (red signal) and dTrpNPs (green signal) acquired using $640 \mathrm{~nm}$ and $560 \mathrm{~nm}$ lasers respectively, after $2.5 \mathrm{~h}, 5 \mathrm{~h}, 8.5 \mathrm{~h}$ and $24 \mathrm{~h}$ observation times. Confocal parameters were adjusted to minimize the intrinsic fluorescence of dTrpNPs under 640 laser scanning. It was observed that for all three organelles i.e., late endosome, early endosome, and lysosome; the colocalization (yellow signal) was maximum until first $5 \mathrm{~h}$ and almost negligible after that. The PCC (Pearson correlation coefficient) values were used to analyze the images and quantify the extent of colocalization (yellow signal) of organelles with dTrpNPs (ESI Table S1). The PCC values estimated at different incubation times indicates the amount of colocalization. Fig. $4 d$ 1-2 and $4 d$ 5-6 and corresponding PCC values (0.5-0.6) suggest that dTrpNPs partially colocalize with both early and late endosome compartments after $2.5 \mathrm{~h}$ and $5 \mathrm{~h}$ observation time. Conversely after $8 \mathrm{~h}$ and $24 \mathrm{~h}$, the confocal microscopic images (Fig. 4d 3-4 and Fig. 4d 7-8) and the corresponding PCC values (0.2-0.3) indicate a weak colocalization of dTrpNPs with both early and late endosome. In addition, negligible colocalization with lysosome at all times was found (Fig. 4d 9-12) indicating lysosomes are not involved in the dTrpNPs trafficking. Taken together, these results clearly suggest that the endosomal escape of dTrpNPs toward the cytosol progressively occurs after $2.5 \mathrm{~h}$ incubation. Subsequently, the shift in $\mathrm{pH}$ from 5.5 to 7 can trigger the dissipative dissolution of dTrpNPs in the cytosol. This agrees with our study in test tube that show that dTrpNPs are dissolving very slowly at the endosomal acid $\mathrm{pH}$ 5-6. A possible mechanism for dTrpNPs endosomal escape could be the "proton sponge effect" depicted in Supplementary Fig. S12. This is typically mediated by species with high buffering capacity in the endosomal "pH change window", such as amine-rich molecules with pKa around 629,30 , which can produce osmotic imbalance inside the endosome and ultimately lead to disruption of endosomal membrane ${ }^{30}$. Indeed, the potentiometric titration curve of dTrp (ESI Fig.S8), shows its buffering capacity in the biological relevant region, between $\mathrm{pH}$ 5 and 7 because of protonation/deprotonation of aromatic hydroxyl groups. Hence, the endosomal escape of the dTrpNPs could be potentially be mediated by "proton sponge effect" triggered by dTrpNPs.

In conclusion, our results show that the dTrpNPs provide a multispectral bioimaging tool suitable to track their intracellular fate. dTrpNPs are promptly internalized in MDA-MB-231 cells, trafficked from early to late endosome, released into the cytosol by escaping the endosomes and dissolved in the neutral cytosolic environment. It is worth noting that apart from $\mathrm{pH}$, in the intracellular milieu dTrpNPs may also experience alternative experimental condition which may induce the disassembling of the aggregates. Competitive interaction with lipids or associating protein with dTrp could potentially also promote the dissolution of dTrpNPs in the cytosol.

\section{Therapeutic properties of dTrpNPs}

We finally sought to investigate possible applications of dTrpNPs in the biomedical field. For instance, Fig. S13 show that dTrpNPs possess high antioxidant and radical scavenging properties. Therefore, dTrpNPs can potentially be used to protect cells against any oxidative damage. Furthermore, the applicability of dTrpNP as a platform for sustained anticancer drugs delivery was also considered. The sustained release of drugs from nanoparticles fuelled by the cytosolic $\mathrm{pH}$ can be advantageous to attain intracellular therapeutic concentrations with higher selectivity and specificity.

Doxorubicin, DOX, was incubated with dTrpNPs at pH 5-6 and a high loading efficiency ( $70 \%$ with $0.38 \mathrm{mg}$ DOX/1 mg of dTrp) was observed. The high loading capacity can be attributed to the electrostatic and hydrophobic interaction between dTrpNPs and DOX. The release of DOX was studied in PBS ( $\mathrm{pH} \mathrm{7.4)} \mathrm{at} 37$ ${ }^{\circ} \mathrm{C}$ by monitoring the intensity of fluorescence emission spectra at $\lambda_{\text {ex }}=480 \mathrm{~nm}$. Fig. $5 \mathrm{a}$ shows the percentage release of the doxorubicin as a function of time. The nanoparticles show slow and bimodal release profile of the drug where, initial release could be due to some adsorbed drug on to the surface of dTrpNP. The surge in the release of the drug after $10 \mathrm{~h}$ could be due to the erosion or dissolution of dTrpNP. Fig. 5b illustrates the MDA-MB-231 viability when treated with DOX and DOX loaded dTrpNP (dTrpNP+DOX) as a function of different concentration of DOX ranging from 0.19 to $12 \mu \mathrm{g} / \mathrm{ml}$ after $24 \mathrm{~h}$ and $48 \mathrm{~h}$. These data show that the cell viability decreases with an increase in the concentration of DOX. The toxicity of dTrpNP +DOX was less than that of free DOX after $24 \mathrm{~h}$ due to the slower release of DOX from the nanoparticles. However, the cytotoxicity of dTrpNP+DOX was comparable with that of free 
DOX after 48 h. Fig. 5c shows the confocal microscopy images of the cells incubated with dTrpNP+DOX particles at different incubation times $2 \mathrm{~h}$ and $24 \mathrm{~h}$. The intrinsic green fluorescence
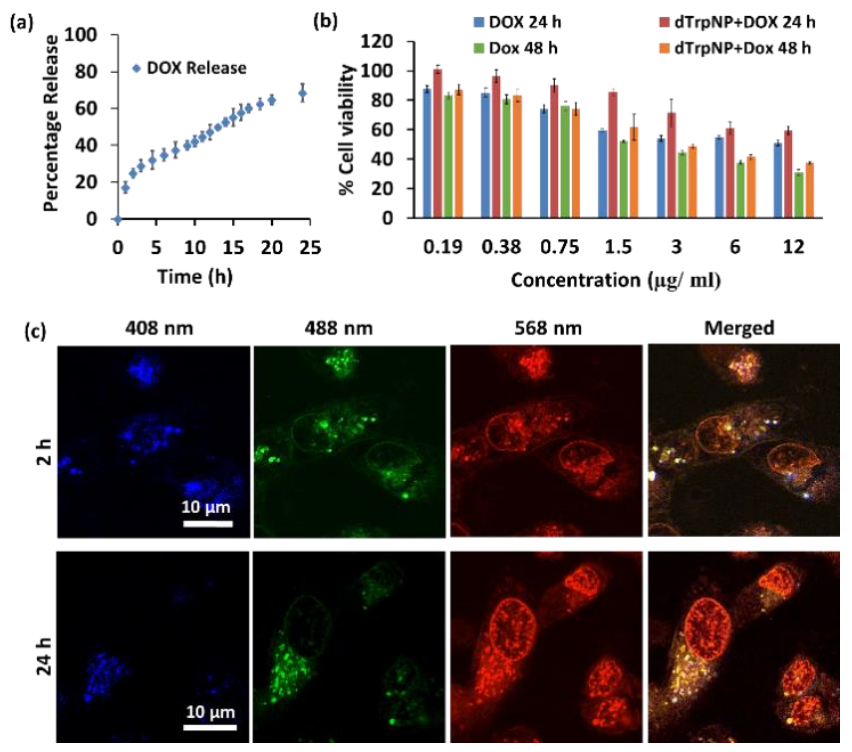

Figure 5: Intracellular release of DOX by dTrpNPs (a) The percentage release of DOX from the $d T r p N P+D O X$ (Blue) dissolution in $P B S(p H=7.4)$ at $37{ }^{\circ} \mathrm{C}$ as a function of time. (b) Cell viability evaluation of DOX and dTrpNP +DOX at different concentrations of DOX after 24 and 48 hours towards MDA-MB 231 cells. (c) Confocal microscopic images of the MDA-MB231 cells incubated with dTrpNP $+D O X$ for 2 and $24 h$ showing the release of DOX from nanoparticles into nucleus.

signal arising from dTrpNP remain confined in the cytosol whereas an increase in red fluorescence as a function of time is observed in the nucleus. This clearly indicates the slow accumulation of doxorubicin into the nucleus upon release from dTrpNP. The accumulation of doxorubicin into the nucleus is a clear evidence of release of the drug from dTrpNP. The drug can be released as a result of the disassembly of dTrpNP or because of diffusion across the nanoparticles. On the other hand, free DOX at the same concentration can completely accumulate into the nucleus just after 1-2 $\mathrm{h}$ of incubation as shown in the Fig. S14. Overall this study suggests that the dTrpNP can be successfully used to attain the controlled release of the drug so that the required concentration of the drug can be maintained within therapeutic levels over extended period of time.

\section{Conclusion}

In conclusion we have presented computational and experimental approaches to demonstrate the role of the acoustic field in the formation of out-of-equilibrium nanoaggregates using a simple amino acid, L-tryptophan, as a building block. The collapse of acoustic cavitation bubble first triggered the formation of tryptophan dimers and then ultimately acted as a fuel for dissipative self-assembly of tryptophan dimers to form uniform nanostructures with multifarious properties. The characteristic optical and biofunctional properties of synthesized nanoparticles made them a powerful tool for sustained release of anti-cancer drugs and for probing their intracellular trafficking. In addition, the insight provided in this work paved the way for the ultrasound driven dissipative self-assembly applicable on set of biomolecules which are currently under investigation. An alternative strategy to pursue is the rational design of ad-hoc building blocks combining experiments, simulation and acoustic theory to predict the ultrasonic driven self-assembly.

\section{Conflicts of interest}

There are no conflicts to declare

\section{Acknowledgements}

This work was supported by the Australian Research Council (ARC) under a Future Fellowship (F. Cavalieri, FT140100873) anf the University of Melbourne Establishment Grant (F. Cavalieri). This work received funding from the European Union's Horizon 2020 research and innovation programme under the Marie SkłodowskaCurie grant agreement No. 690901 (NANOSUPREMI). This work was performed in part at the Materials Characterization and Fabrication Platform (MCFP) at The University of Melbourne. We acknowledge the University of Melbourne for their support through MRS scholarship and Norma Hilda Schuster Scholarship (S.K. Bhangu). We also thank the CINECA consortium for providing computational resources. Special Thanks to Mr. Yang Shen for his contribution with bubble dynamics studies.

\section{Notes and references}

1. E. Reisler and E. H. Egelman, J. Biol. Chem, 2007, 282, 36133-36137.

2. S. A. van Rossum, M. Tena-Solsona, J. H. van Esch, R. Eelkema and J. Boekhoven, Chem. Soc. Rev., 2017, 46, 5519-5535.

3. S. De and R. Klajn, Adv. Mater., 2018, 30, 1706750.

4. G. Ragazzon and L. J. Prins, Nat. nanotechnol, 2018, 13, 882-889

5. A. Sorrenti, J. Leira-Iglesias, A. J. Markvoort, T. F. de Greef and T. M. Hermans, Chem. Soc. Rev., 2017, 46, 5476-5490.

6. G. Ragazzon, M. Baroncini, S. Silvi, M. Venturi and A. Credi, Nat. nanotechnol, 2015, 10, 70.

7. E. Mattia, S. Otto. Nat. nanotechnol. 2015, 10, 111.

8. F. Rakotondradany, M. Whitehead, A. M. Lebuis and H. F. Sleiman, Chem. Eur. J. 2003, 9, 4771-4780.

9. J. Boekhoven, A. M. Brizard, K. N. Kowlgi, G. J. Koper, R. Eelkema and J. H. van Esch, Angew Chem, 2010, 122, 49354938.

10. J. Boekhoven, A. M. Brizard, K. N. Kowlgi, G. J. Koper, R. Eelkema and J. H. van Esch, Angew Chem Int Ed, 2010, 49, 4825-4828.

11. E. Del Grosso, A. Amodio, G. Ragazzon, L. J. Prins and F. Ricci, Angew Chem Int Ed, 2018, 57, 10489-10493. 
12. K. Nakajima, H. Ogi, K. Adachi, K. Noi, M. Hirao, H. Yagi and Y. Goto, Scientific reports, 2016, 6, 22015.

13. C. G. Pappas, T. Mutasa, P. W. J. M. Frederix, S. Fleming, S. Bai, S. Debnath, S. M. Kelly, A. Gachagan and R.V. Ulijn, Mater. Horiz., 2015, 2, 198-202.

14. F. Cavalieri, E. Colombo, E. Nicolai, N. Rosato and M. Ashokkumar, Mater. Horiz., 2016, 3, 563-567.

15. S. K. Bhangu and M. Ashokkumar, in Sonochemistry, Springer, 2017, 1-28.

16. K. Tao, Z. Fan, L. Sun, P. Makam, Z. Tian, M. Ruegsegger, S. Shaham-Niv, D. Hansford, R. Aizen, Z. Pan, and S. Galster, Nat. comm, 2018, 9, 3217.

17. J. I. Mujika, J. Uranga and J. M. Matxain, Chem. Eur. J. 2013, 19, 6862-6873.

18. D. Sunartio, M. Ashokkumar and F. Grieser, J. Am. Chem. Soc., 2007, 129, 6031-6036.

19. K. Yasui, in Acoustic Cavitation and Bubble Dynamics, Springer, 2018, pp. 37-97.

20. Y. Shen, K. Yasui, T. Zhu and M. Ashokkumar, Phys. Chem. Chem. Phys, 2017, 19, 20635-20640.

21. L. Carroll, D. I. Pattison, J. B. Davies, R. F. Anderson, C. Lopez-Alarcon and M. J. Davies, Free Radic. Biol. Med, 2017, 113, 132-142.

22. V. Paviani, G. T. Galdino, J. N. d. Prazeres, R. F. Queiroz and O. Augusto, J. Braz. Chem. Soc., 2018, 29, 925-933.

23. A. Farrotti, P. Conflitti, S. Srivastava, J. K. Ghosh, A. Palleschi, L. Stella and G. Bocchinfuso, Molecules, 2017, 22, 1235.

24. G. Bocchinfuso, C. Mazzuca, P. Conflitti, D. Cori, T. Coviello and A. Palleschi, Z. Phys. Chem., 2016, 230, 1395-1410.

25. M. Levitt and M. F. Perutz, J. Mol. Biol., 1988, 201, 751-754.

26. C. Eggeling, J. Widengren, R. Rigler and C. Seidel, Anal. Chem., 1998, 70, 2651-2659.

27. H. NamáChan, Chem Comm, 2014, 50, 13578-13580.

28. Y. Q. Sun, J. Liu, X. Lv, Y. Liu, Y. Zhao and W. Guo, Angew. Chem. Int. Ed., 2012, 51, 7634-7636.

29 D. W. Pack, D. Putnam and R. Langer, Biotechnol. Bioeng, 2000, 67, 217-223.

30. C. Lin and J. F. Engbersen, J.Control. Release, 2008, 132, 267-272.

For Graphical Abstract

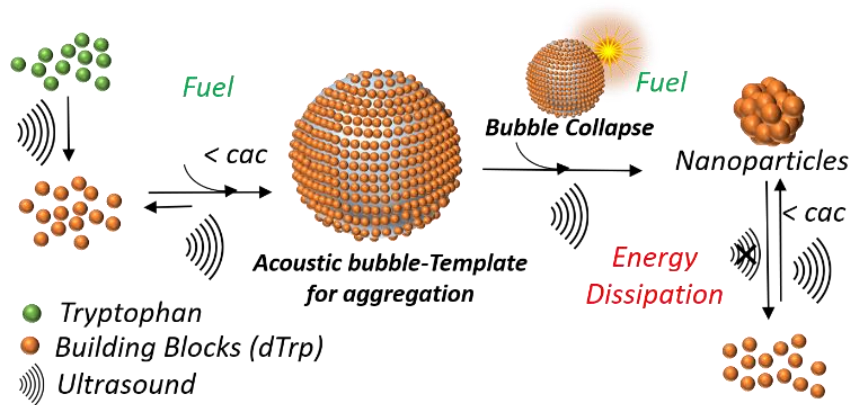

The out-of-equilibrium self-assembly of simple aromatic amino acid molecules is driven by high frequency ultrasound, where acoustic energy acts as a fuel to form uniform supramolecular nanostructures with multifarious optical and biological properties. 


\section{University Library}

\section{- M M I E E R VA A gateway to Melbourne's research publications}

Minerva Access is the Institutional Repository of The University of Melbourne

Author/s:

Bhangu, SK;Bocchinfuso, G;Ashokkumar, M;Cavalieri, F

Title:

Sound-driven dissipative self-assembly of aromatic biomolecules into functional nanoparticles

Date:

2020-03-01

Citation:

Bhangu, S. K., Bocchinfuso, G., Ashokkumar, M. \& Cavalieri, F. (2020). Sound-driven dissipative self-assembly of aromatic biomolecules into functional nanoparticles. Nanoscale Horizons, 5 (3), pp.553-563. https://doi.org/10.1039/c9nh00611g.

Persistent Link:

http://hdl.handle.net/11343/241814 